\title{
Predictor-Based Compensation for Electromechanical Delay During Neuromuscular Electrical Stimulation
}

\author{
Nitin Sharma, Chris M. Gregory, and Warren E. Dixon, Member, IEEE
}

\begin{abstract}
Electromechanical delay (EMD) is a biological artifact that arises due to a time lag between electrical excitation and tension development in a muscle. EMD is known to cause degraded performance and instability during neuromuscular electrical stimulation (NMES). Compensating for such input delay is complicated by the unknown nonlinear muscle force-length and muscle force-velocity relationships. This paper provides control development and a mathematical stability analysis of a NMES controller with a predictive term that actively accounts for EMD. The results are obtained through the development of a novel predictor-type method to address the delay in the voltage input to the muscle. Lyapunov-Krasovskii functionals are used within a Lyapunov-based stability analysis to prove semi-global uniformly ultimately bounded tracking. Experiments on able-bodied volunteers illustrate the performance and robustness of the developed controller during a leg extension trajectory following task.
\end{abstract}

Index Terms-Electromechanical delay, functional electrical stimulation, input delay, Lyapunov-Krasovskii functionals, neuromuscular electrical stimulation (NMES), nonlinear control.

\section{INTRODUCTION}

$\mathbf{R}$ EHABILITATION through neuromuscular electrical stimulation (NMES) (also called functional electrical stimulation (FES) for specific functional tasks) can be applied as a treatment to improve the activities of daily living for some physically impaired individuals. These individuals include persons who have completely absent or limited functional control of limbs or organs due to the impeded neural communication with central nervous system and/or brain (e.g., some types of spinal cord injury, traumatic brain injury, multiple sclerosis, stroke) [1]. Provided the peripheral nervous system is not harmed during the original disease or primary injury, NMES can be used as an artificial application of electrical potential across a muscle group as a treatment to rehabilitate or revive functionality. NMES has the potential to be prescribed as a treatment for advanced functional tasks (e.g., gait retraining) if the resulting limb motion could be accurately and precisely controlled. Based on this motivation,

Manuscript received January 21, 2011; revised June 03, 2011 and July 22, 2011; accepted July 26, 2011. Date of publication October 03, 2011; date of current version December 07, 2011. This work was supported in part by the NSF CAREER Award CMS-0547448.

N. Sharma is with the Department of Physiology, University of Alberta, Edmonton, AB, T6G 2E1 Canada (e-mail: nitin2@ualberta.ca).

C. M. Gregory is with the Department of Health Sciences and Research, Medical University of South Carolina, Charleston, SC 29425 USA (e-mail: gregoryc@musc.edu).

W. E. Dixon is with the Department of Mechanical and Aerospace Engineering, University of Florida, Gainesville, FL 32611 USA (e-mail: wdixon@ufl.edu).

Color versions of one or more of the figures in this paper are available online at http://ieeexplore.ieee.org.

Digital Object Identifier 10.1109/TNSRE.2011.2166405 researchers have developed various closed-loop strategies (c.f. [2]-[14] and the references therein). Recently, efforts have been made to develop nonlinear control techniques [5], [15]-[19] to obtain improved error performance and/or asymptotic tracking [5], [17], [18] (proven through a closed-loop stability analysis), even in the presence of bounded exogenous disturbances [17], [18]. However, several open challenges persist due to the uncertain nonlinear musculoskeletal dynamics, time-dependent muscle force reduction due to fatigue, unmodeled disturbances such as muscle spasticity or external changes in muscle loads, delayed muscle response, etc. In particular, this paper focuses on the development of a NMES control method to actively compensate for electromechanical delay (EMD) in the muscle response.

EMD is a function of a number of phenomena including: finite propagation time of the chemical ions in the muscle, crossbridge formation between actin-myosin filaments, stretching of the series elastic components in response to the external electrical input, synaptic transmission delays, and others [20]-[22]. In [6], EMD is defined as the electromechanical response time that represents the time difference between the moment when the electromyogram (EMG) signal is generated and the moment when the muscle force occurs. In results such as [5], [15], and the current result, the EMD is modeled as an input delay in the musculoskeletal dynamics. Input delay can cause performance degradation and has also been reported to potentially cause instability during human stance experiments [23]. Despite the fact that EMD is exhibited in muscle response and can lead to instability, no NMES controller has been developed to actively compensate for this phenomena. Previous results such as [6] and [23]-[25] examine EMD effects by implementing a standard PD controller during stance (or quiet standing) experiments that show robustness to the delays. The controllers in such results are not modified to include a delay compensation term. These results have no mathematical proof of stability when the plant has uncertainties, nonlinearities, and/or disturbances. This is in contrast to the development in this paper which is later described in the introduction and the subsequent sections as the "PD controller with delay compensation". This controller actively compensates for the EMD using the predictor-based term and is mathematically shown to achieve an uniformly ultimately bounded tracking for an uncertain nonlinear musculoskeletal system with additive disturbance.

Various methods exist in the general time-delay control literature to compensate for actuator or input delay, but the existing approaches such as Smith predictor methods [26], Artstein model reduction [27], finite spectrum assignment [28], and continuous pole placement [29] are applicable to only linear plants. The control problem becomes especially complicated 
when parametric uncertainties, nonlinearity, and additive disturbances are considered. Adaptive Posicast Controllers have also been developed for uncertain linear systems with delays and for automotive applications in [30]-[32]. The few approaches that have been developed for input delayed nonlinear systems include modified Smith Predictor-based control methods and passivity-based architectures for state synchronization [33]-[36]. These techniques either require or assume a known nonlinear plant model or an adaptive estimate of the nonlinear plant. In [37] and [38], approaches are provided to construct LyapunovKrasovskii (LK) functionals for stabilizing input delayed nonlinear system in feedback form and nonlinear cascade systems, where the delay can enter the system through the input or the states. The work in [39]-[41] provide fundamental contributions to the input delay problem in feedforward systems, but its applicability to general uncertain mechanical systems modeled by Euler-Lagrange dynamics (e.g., a musculoskeletal dynamics driven by NMES ) is not clear. A method to transform a cart-pendulum system (a typical example of Euler-Lagrange system) to a feedforward system and subsequently to design a tracking controller was provided in [42]. These transformations require exact model knowledge, thus the technique is not applicable when the system parameters are unknown or dynamics are uncertain, which implies that methods developed for feedforward systems with delay may not be applicable to uncertain musculoskeletal dynamics. In [43] and [44], a backstepping approach that utilizes ODE-PDE cascade transformation for input delayed systems is extended to nonlinear control systems with an actuator delay of unrestricted length. The results in [45]-[47] utilize controllers developed for delay-free systems and prove robustness to the delay provided certain delay dependent conditions hold true. Also, these results only establish local exponential or asymptotic stability for a zero/stationary solution of uncertain nonlinear systems (linearized about a setpoint). Unlike the current paper that utilizes a predictor-based method to compensate for the input delay in uncertain musculoskeletal system (without linearization), prior results lack applicability as a stabilizing control for an input delayed musculoskeletal system or any nonlinear plant with parametric uncertainty and/or additive bounded disturbances.

Recently, a predictor-based control method was developed for an uncertain input delayed system with additive disturbances [48]. This result suggests that a PD/PID controller can be augmented with a delay compensator that contains a finite integral of past control values to transform the delayed system into a delay-free system. Building on the results in [48], this paper focuses on the development of a tracking controller for a nonlinear musculoskeletal system with input delay, where the time delay is assumed to be a known constant. The dynamics include parametric uncertainty and additive bounded disturbances. The control design and stability analysis yields a PD controller with an augmented predictor component. The key contributions of this effort are the design of a delay compensating auxiliary signal to obtain a time delay free open-loop error system and the construction of a LK functional to cancel the time delayed terms. An LK functional containing a finite integral of control input values is used in a Lyapunov-based analysis that proves the tracking error is semi-global uniformly

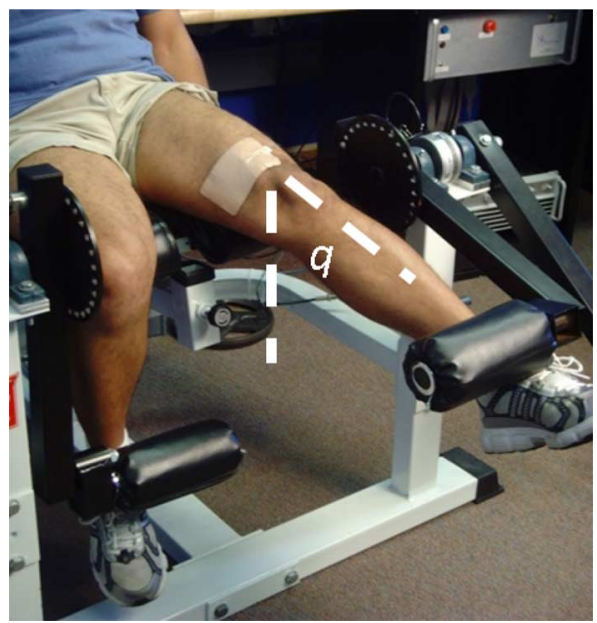

Fig. 1. Experimental test bed: modified leg extension machine. Knee angle $q(t)$ is measured with respect to the vertical reference line.

ultimately bounded. Two sets of experiments were performed on able-bodied individuals. The first set of experiments was performed to characterize input delay in NMES with respect to voltage amplitude, and the second set of experiments highlights the added value of including the delay compensating term in the control design.

\section{Muscle Activation AND Limb Model}

The uncertain nonlinear musculoskeletal dynamics for the torque about the one-degree of rotational freedom knee-joint for a person using a leg extension machine (see Fig. 1) is given as [5], [18]

$$
M_{I}+M_{e}+M_{g}+M_{v}+d=u(t-\tau)
$$

In (1), $M_{I}(\ddot{q}) \in \mathbb{R}$ denotes the inertial force about the knee-joint axis, $M_{e}(q) \in \mathbb{R}$ denotes the elastic torque due to joint stiffness, $M_{g}(q) \in \mathbb{R}$ denotes the gravitational moment, $M_{v}(\dot{q}) \in \mathbb{R}$ denotes the viscous torque due to damping in the musculotendon complex, $d(t) \in \mathbb{R}$ represents unknown unmodeled bounded disturbances, and $u(t-\tau) \in \mathbb{R}$ denotes the delayed torque production at the knee joint, where $\tau \in \mathbb{R}^{+}$denotes the EMD. The inertia and gravitational effects in (1) are modeled as

$$
M_{I}(\ddot{q})=J \ddot{q}, \quad M_{g}(q)=m g l \sin (q)
$$

where $q(t), \dot{q}(t), \ddot{q}(t) \in \mathbb{R}$ denote the angular position, velocity, and acceleration of the lower shank about the knee-joint, respectively, $J \in \mathbb{R}^{+}$denotes the unknown inertia of the combined human shank-foot and machine, $m \in \mathbb{R}^{+}$denotes the unknown combined mass of the shank-foot and machine, $l \in \mathbb{R}^{+}$is the unknown distance between the knee-joint and the lumped center of mass of the leg-machine system, and $g \in \mathbb{R}^{+}$denotes the gravitational acceleration. The elastic force is modeled as [49]

$$
M_{e}(q)=k_{1}\left(\exp \left(-k_{2} q\right)\right)\left(q-k_{3}\right)
$$


where $k_{1}, k_{2}, k_{3} \in \mathbb{R}^{+}$are unknown coefficients. The viscous moment $M_{v}(\dot{q})$ is modeled as [5]

$$
M_{v}(\dot{q})=B_{1} \tanh \left(-B_{2} \dot{q}\right)-B_{3} \dot{q}
$$

where $B_{1}, B_{2}$, and $B_{3} \in \mathbb{R}^{+}$are unknown constants.

The torque produced about the knee is controlled through muscle forces that are elicited by NMES. For simplicity (and without loss of generality), the subsequent development focuses on producing knee torque through muscle tendon forces generated by electrical stimulation of the quadriceps (i.e., antagonistic muscle forces are not considered). The total muscle force is a net sum of active force generated by contractile, elastic, and viscous elements [18]. The muscle force generated at the tendon is the projection of net sum of these elements along the line parallel to the tendon. The force development in the muscle is delayed due to the finite propagation time of chemical ions such as $\mathrm{Ca}^{2+}$ and action potential along the $\mathrm{T}$-tubule system, cross-bridge formation between actin and myosin filaments, the subsequent tension development, and the stretching of the series elastic components by the contractile components in the muscle [20]-[22] The EMD is influenced by factors such as fatigue, rate of force production, and types of muscle contractions. The total delayed muscle force generated at the tendon, denoted by $F(t-\tau) \in \mathbb{R}$, is defined as

$$
F(t-\tau)=\eta(q, \dot{q}) V(t-\tau)
$$

In (4), $\eta(q, \dot{q}) \in \mathbb{R}^{+}$denotes an unknown nonlinear function of the muscle length and velocity, and the applied voltage potential across the quadriceps muscle, denoted by $V(t-\tau) \in \mathbb{R}$, includes the time delay to capture the latency that is present between the application of voltage and force production [5], [15]. The introduction of the unknown nonlinear function $\eta(q, \dot{q})$ enables the muscle contraction to be considered under general dynamic conditions in the subsequent control development. The uncertain and unknown function $\eta(q, \dot{q})$ captures the dynamic characteristics of muscle recruitment (approximated by a continuously differentiable function), muscle force-length and muscle force-velocity relationships, and active and passive muscle characteristics [18], [19]. The knee torque is related to the muscle tendon force as

$$
u(t-\tau)=\zeta(q(t)) F(t-\tau)
$$

where $\zeta(q(t)) \in \mathbb{R}^{+}$denotes a positive moment arm that changes with the extension and flexion of the leg [50], [51].

The model developed in (1)-(5) is used to examine the stability of the subsequently developed controller, but the controller does not explicitly depend on these models. The following assumptions and notations are used to facilitate the subsequent control development and stability analysis.

Assumption 1: The moment arm $\zeta(q)$ is assumed to be a nonzero, positive, bounded function [50], [51] whose first two time derivatives exist, and based on the empirical data [52], [53], the function $\eta(q, \dot{q})$ is assumed to be a nonzero, positive, and bounded function with a bounded first and second time derivatives.
Assumption 2: The auxiliary nonzero unknown scalar function $\Omega(q, \dot{q}) \in \mathbb{R}^{+}$is defined as

$$
\Omega=\zeta \eta
$$

where the first and second time derivatives of $\Omega(q, \dot{q})$ are assumed to exist and be bounded (see Assumption 1).

Assumption 3: The unknown disturbance $d(t)$ is bounded and its first and second derivatives with respect to time exist and are bounded. Based on Assumptions 1 and 2, the ratio $d(t) / \Omega(q, \dot{q})$ denoted as $d_{\Omega 2}(q, \dot{q}, t)$ is also assumed to be bounded and its first and second derivatives with respect to time exist and are bounded.

Assumption 4: Based on Assumption 1, the ratio $J / \Omega(q, \dot{q})$, denoted as $J_{\Omega}(q, \dot{q}) \in \mathbb{R}^{+}$, can be upper bounded as

$$
J_{1} \leq\left|J_{\Omega}\right| \leq J_{2}
$$

where $J_{1}, J_{2} \in \mathbb{R}^{+}$are known constants.

Assumption 5: Since the scope of this initial development involves only one type of dynamic contraction and constant stimulation frequency, the input delay $\tau$ is assumed to be a known constant. This assumption is reasonable based on the data provided in the subsequent experimental results section that quantifies the EMD under a specific set of conditions (e.g., muscle fatigue effects are not considered). However, the development of a controller that does not require this assumption is an open theoretical challenge that is the focus of future efforts.

Notation: A delayed state in the subsequent development and analysis is denoted as $x(t-\tau)$ or as $x_{\tau}$ while a nondelayed state is denoted as $x(t)$ or as $x$.

\section{Control Development}

\section{A. Objective}

The objective is to develop a controller that will enable the knee angle of the input delayed musculoskeletal dynamics in (1) to track a desired trajectory, denoted by $q_{d}(t) \in \mathbb{R}$. A tracking error, denoted by $e_{1}(t) \in \mathbb{R}$, is defined to quantify the objective as

$$
e_{1}=q_{d}-q
$$

To facilitate the subsequent design and stability analysis, an auxiliary error signal $e_{2}(t) \in \mathbb{R}$ is defined as

$$
e_{2}=\dot{e}_{1}+\alpha e_{1}-B \int_{t-\tau}^{t} V(\theta) d \theta
$$

where $\alpha, B \in \mathbb{R}^{+}$are known constants (control gains). The unknown force-length, force-velocity, and moment arm relationships, represented by auxiliary function $\Omega(q, \dot{q})$ in (6), and the EMD present challenges for the subsequent control design. To facilitate the subsequent stability analysis, the error between $B$ and $J_{\Omega}^{-1}(q, \dot{q})$ is defined by

$$
\xi=B-\frac{\Omega}{J}
$$


where $\xi(q, \dot{q}) \in \mathbb{R}$ satisfies the following:

$$
|\xi| \leq \bar{\xi}
$$

where $\bar{\xi} \in \mathbb{R}^{+}$denotes a known constant.

The open-loop tracking error system can be developed by multiplying the time derivative of (9) by $J_{\Omega}(q, \dot{q})$ and utilizing the expressions in (1), (8), and (10) to obtain

$$
\begin{aligned}
J_{\Omega} \dot{e}_{2}=J_{\Omega} \ddot{q}_{d}+M_{e \Omega}+ & M_{g \Omega}+M_{v \Omega}+d_{\Omega} \\
& +\alpha J_{\Omega} \dot{e}_{1}-V-J_{\Omega} \xi\left[V-V_{\tau}\right]
\end{aligned}
$$

where nonlinear functions $M_{e \Omega}(q, \dot{q}), M_{g \Omega}(q, \dot{q})$, and $M_{v \Omega}(q, \dot{q})$ are defined as

$$
M_{e \Omega}=\frac{M_{e}}{\Omega}, \quad M_{g \Omega}=\frac{M_{g}}{\Omega}, \quad M_{v \Omega}=\frac{M_{v}}{\Omega} .
$$

Let $N_{d}\left(q_{d}, \dot{q}_{d}, \ddot{q}_{d}, t\right) \in \mathbb{R}$ be defined as

$$
N_{d}=J_{\Omega d} \ddot{q}_{d}+M_{v \Omega d}+M_{g \Omega d}+M_{e \Omega d}
$$

where the notation $J_{\Omega d}\left(q_{d}, \dot{q}_{d}\right), \quad M_{v \Omega d}\left(q_{d}, \dot{q}_{d}\right)$, $M_{g \Omega d}\left(q_{d}, \dot{q}_{d}\right), M_{e \Omega \Omega d}\left(q_{d}, \dot{q}_{d}\right)$, represents $J_{\Omega}(q, \dot{q}), M_{v \Omega}(q, \dot{q})$, $M_{g \Omega}(q, \dot{q}), M_{e \Omega}(q, \dot{q})$ expressed in terms of desired limb position and velocity. Based on (12) and the subsequent stability analysis, the voltage input $V\left(e_{2}\right) \in \mathbb{R}$ is designed as

$$
V=k_{b} e_{2}
$$

where $k_{b} \in \mathbb{R}^{+}$is a known control gain that can be expanded as

$$
k_{b}=k_{b_{1}}+k_{b_{2}}+k_{b_{3}}
$$

to facilitate the subsequent analysis, where $k_{b_{1}}, k_{b_{2}}$, and $k_{b_{3}} \in$ $\mathbb{R}^{+}$are known constants. The closed-loop error system is determined by adding and subtracting $N_{d}\left(q_{d}, \dot{q}_{d}, \ddot{q}_{d}, t\right) \in \mathbb{R}$ to (12) and using (9) and (13) as

$$
J_{\Omega} \dot{e}_{2}=-\frac{1}{2} \dot{J}_{\Omega} e_{2}+\tilde{N}+S-e_{1}-k_{b} e_{2}-k_{b} J_{\Omega} \xi\left[e_{2}-e_{2 \tau}\right]
$$

where the auxiliary terms $\tilde{N}\left(e_{1}, e_{2}, t, \tau\right), N\left(e_{1}, e_{2}, t, \tau\right)$, $S\left(q, \dot{q}, q_{d}, \dot{q}_{d}, \ddot{q}_{d}, t\right) \in \mathbb{R}$ are defined as

$N=\frac{1}{2} \dot{J}_{\Omega} e_{2}+J_{\Omega} \ddot{q}_{d}+M_{e \Omega}+M_{v \Omega}+M_{g \Omega}+\alpha J_{\Omega} e_{2}-\alpha^{2} J_{\Omega} e_{1}$

$$
+e_{1}+\alpha J_{\Omega} B \int_{t-\tau}^{t} V(\theta) d \theta
$$

$\tilde{N}=N-N_{d}, \quad S=N_{d}+d_{\Omega}$

where $\tilde{N}\left(e_{1}, e_{2}, t, \tau\right)$ and $S\left(q, \dot{q}, q_{d}, \dot{q}_{d}, \ddot{q}_{d}, t\right)$ can be upper bounded as

$$
\tilde{N} \leq \rho(\|z\|)\|z\|, \quad\|S\| \leq \varepsilon
$$

In (17), $\varepsilon \in \mathbb{R}^{+}$is a known constant, the bounding function $\rho(\|z\|) \in \mathbb{R}$ is a positive globally invertible nondecreasing function, and $z\left(e_{1}, e_{2}, e_{z}\right) \in \mathbb{R}^{3}$ is defined as

$$
z=\left[\begin{array}{lll}
e_{1} & e_{2} & e_{z}
\end{array}\right]^{T}
$$

where $e_{z}(V) \in \mathbb{R}$ is defined as

$$
e_{z}=\int_{t-\tau}^{t} V(\theta) d \theta
$$

Based on (15) and the subsequent stability analysis, LK functionals $P(V), Q\left(e_{2}\right) \in \mathbb{R}$ are defined as

$$
\begin{aligned}
& P=\omega \int_{t-\tau}^{t}\left(\int_{s}^{t} V(\theta)^{2} d \theta\right) d s \\
& Q=\frac{\bar{\xi} J_{2} k_{b}}{2} \int_{t-\tau}^{t} e_{2}(\theta)^{2} d \theta
\end{aligned}
$$

where $\omega \in \mathbb{R}^{+}$is a known constant.

Theorem 1: The controller given in (13) ensures semi-global uniformly ultimately bounded tracking

$$
\left|e_{1}(t)\right| \leq \epsilon_{0} \exp \left(-\epsilon_{1} t\right)+\epsilon_{2}
$$

where $\epsilon_{0}, \epsilon_{1}, \epsilon_{2} \in \mathbb{R}^{+}$denote constants, provided the control gains $\alpha$ and $k_{b}$ introduced in (9) and (13), respectively are selected according to the sufficient conditions

$$
\alpha>\frac{B^{2} \tau}{2 \omega}, \quad k_{b_{3}}>\frac{2 \bar{\xi} J_{2}\left(k_{b_{1}}+k_{b_{2}}\right)+\omega k_{b}^{2} \tau}{1-2 \bar{\xi} J_{2}}
$$

where the known positive constants $J_{2}, B, \bar{\xi}, \omega$ are defined in (7), (9), (11), and (19), respectively.

Remark 1: The second sufficient gain condition in (21) can be satisfied by selecting $\omega, k_{b_{1}}$, and $k_{b_{2}}$ sufficiently small and $k_{b_{3}}$ sufficiently large, provided $1-2 \vec{\xi} J_{2}>0$. The condition that $1-2 \bar{\xi} J_{2}>0$ indicates that the constant approximation matrix $B$ must be chosen sufficiently close to $\Omega(q, \dot{q}) / J$ so that $|B-(\Omega(q, \dot{q}) / J)|<\left(1 / 2 J_{2}\right)$. Experimental results illustrate the performance/robustness of the developed controller with respect to the estimate mismatch.

Proof: Let $y(t) \in \mathcal{D} \subset \mathbb{R}^{4}$ be

$$
y \triangleq\left[\begin{array}{llll}
e_{1} & e_{2} & \sqrt{P} & \sqrt{Q}
\end{array}\right]^{T} .
$$

A positive definite Lyapunov functional candidate $U(y, t): \mathcal{D} \times$ $[0 \propto) \rightarrow \mathbb{R}$ is defined as

$$
U \triangleq \frac{1}{2} e_{1}^{2}+\frac{1}{2} J_{\Omega} e_{2}^{2}+P+Q
$$


and satisfies the following:

$$
\lambda_{1}\|y\|^{2} \leq U \leq \lambda_{2}\|y\|^{2}
$$

where $\lambda_{1}, \lambda_{2} \in \mathbb{R}^{+}$are known constants.

Taking the time derivative of (23) and using (9) and (15) yields

$$
\begin{aligned}
\dot{U}= & -\alpha e_{1}^{2}+B e_{z} e_{1}+\omega \tau V^{2} \\
& +e_{2}\left(S+\tilde{N}-k_{b} e_{2}-k_{b} J_{\Omega} \xi\left(e_{2}-e_{2 \tau}\right)\right) \\
& +\frac{\bar{\xi} J_{2} k_{b}}{2}\left[e_{2}^{2}-e_{2 \tau}^{2}\right]-\omega \int_{t-\tau}^{t} V(\theta)^{2} d \theta
\end{aligned}
$$

where the Leibniz integral rule was applied to determine the time derivative of $P(V)$ and $Q\left(e_{2}\right)$. Using (7) and (17), the terms in (25) can be upper bounded as

$$
\begin{aligned}
\dot{U} \leq & -\alpha e_{1}^{2}-k_{b} e_{2}^{2}+\bar{\xi} J_{2} k_{b} e_{2}^{2}+\omega \tau V^{2}+\left|e_{2}\right| \varepsilon \\
& +\left|e_{2}\right| \rho(\|z\|)\|z\|+B\left|e_{1}\right|\left|e_{z}\right|+\bar{\xi} J_{2} k_{b}\left|e_{2 \tau}\right|\left|e_{2}\right| \\
& +\frac{\bar{\xi} J_{2} k_{b}}{2}\left[e_{2}^{2}-e_{2 \tau}^{2}\right]-\omega \int_{t-\tau}^{t} V(\theta)^{2} d \theta .
\end{aligned}
$$

The following terms in (26) can be upper bounded by utilizing Young's inequality:

$$
\begin{aligned}
B\left|e_{1}\right|\left|e_{z}\right| & \leq \frac{B^{2} \gamma^{2}}{4} e_{1}^{2}+\frac{1}{\gamma^{2}} e_{z}^{2}, \\
\bar{\xi} J_{2} k_{b}\left|e_{2 \tau}\right|\left|e_{2}\right| & \leq \frac{\bar{\xi} J_{2} k_{b}}{2} e_{2}^{2}+\frac{\bar{\xi} J_{2} k_{b}}{2} e_{2 \tau}^{2}
\end{aligned}
$$

where $\gamma \in \mathbb{R}^{+}$is a known constant that is selected as

$$
\gamma>\sqrt{\frac{2 \tau}{\omega}}
$$

Further, by using the Cauchy Schwarz inequality, the following term in (27) can be upper bounded as:

$$
e_{z}^{2} \leq \tau \int_{t-\tau}^{t} V(\theta)^{2} d \theta
$$

After adding and subtracting $\left(\tau / \gamma^{2}\right) \int_{t-\tau}^{t} V(\theta)^{2} d \theta$ to (26), and utilizing (13), (14), (27), and (28), the following expression is obtained:

$$
\begin{aligned}
\dot{U} \leq & -\left(\alpha-\frac{B^{2} \gamma^{2}}{4}\right) e_{1}^{2}-\left(k_{b_{3}}-\omega k_{b}^{2} \tau-2 \bar{\xi} J_{2} k_{b}\right) e_{2}^{2} \\
& -\frac{1}{\tau}\left(\omega-\frac{2 \tau}{\gamma^{2}}\right) e_{z}^{2}-k_{b_{1}} e_{2}^{2}+\rho(\|z\|)\|z\||| e_{2} \mid \\
& -k_{b_{2}} e_{2}^{2}+\left|e_{2}\right| \varepsilon-\frac{\tau}{\gamma^{2}} \int_{t-\tau}^{t} V(\theta)^{2} d \theta
\end{aligned}
$$

By completing the squares, the inequality in (29) can be upper bounded as

$$
\dot{U} \leq-\left\{\beta-\frac{\rho^{2}(\|z\|)}{4 k_{b_{1}}}\right\}\|z\|^{2}-\frac{\tau}{\gamma^{2}} \int_{t-\tau}^{t} V(\theta)^{2} d \theta+\frac{\varepsilon^{2}}{4 k_{b_{2}}}
$$

where $\beta \in \mathbb{R}^{+}$is denoted as

$$
\beta=\min \left(\left(\alpha-\frac{B^{2} \gamma^{2}}{4}\right),\left(k_{b_{3}}-2 \bar{\xi} J_{2} k_{b}-\omega k_{b}^{2} \tau\right), \frac{1}{\tau}\left(\omega-\frac{2 \tau}{\gamma^{2}}\right)\right) .
$$

Since

$$
\begin{aligned}
\int_{t-\tau}^{t}\left(\int_{s}^{t} V(\theta)^{2} d \theta\right) d s & \leq \tau \sup _{s \in[t, t-\tau]}\left[\int_{s}^{t} V(\theta)^{2} d \theta\right] \\
& =\tau \int_{t-\tau}^{t} V(\theta)^{2} d \theta
\end{aligned}
$$

the expression in (30) can rewritten as

$$
\begin{gathered}
\dot{U} \leq-\left\{\beta-\frac{\rho^{2}(\|z\|)}{4 k_{b_{1}}}\right\}\|z\|^{2}-\frac{\tau}{2 \gamma^{2}} \int_{t-\tau}^{t} V(\theta)^{2} d \theta \\
-\frac{1}{2 \gamma^{2}} \int_{t-\tau}^{t}\left(\int_{s}^{t} V(\theta)^{2} d \theta\right)+\frac{\varepsilon^{2}}{4 k_{b_{2}}} .
\end{gathered}
$$

Using the definitions of $z(t)$ in (18), $y(t)$ in (22), and $u(t)$ in (13), the expression in (31) can be upper bounded as

$$
\dot{U} \leq-\bar{\beta}\|y\|^{2}-\left\{\beta-\frac{\rho^{2}(\|z\|)}{4 k_{b_{1}}}\right\}\left\|e_{z}\right\|^{2}+\frac{\varepsilon^{2}}{4 k_{b_{2}}}
$$

where $\bar{\beta}(\|z\|) \in \mathbb{R}^{+}$is

$$
\delta \leq \bar{\beta}=\min \left[\left(\beta-\frac{\rho^{2}(\|z\|)}{4 k_{b_{1}}}\right), \frac{k_{b} \tau}{\gamma^{2} \bar{\xi} J_{2}}, \frac{1}{2 \omega \gamma^{2}}\right]
$$

for some $\delta \in \mathbb{R}^{+}$. By further utilizing (24), the inequality in (32) can be written as

$$
\dot{U} \leq-\frac{\delta}{\lambda_{2}} V+\frac{\varepsilon^{2}}{4 k_{b_{2}}}
$$

Consider a set $\mathcal{S}$ defined as

$$
\mathcal{S} \triangleq\left\{z(t) \in \mathbb{R}^{3} \mid\|z\|<\rho^{-1}\left(2 \sqrt{\beta k_{b_{1}}}\right)\right\}
$$

For $z(0) \in \mathcal{S}$, the linear differential equation in (33) can be solved as

$$
U \leq U(0) e^{-\frac{\delta}{\lambda 2} t}+\frac{\varepsilon^{2} \lambda_{2}}{4 k_{b_{2}} \delta}\left[1-e^{-\frac{\delta}{\lambda_{2}} t}\right]
$$


provided the control gains $\alpha$ and $k_{b}$ are selected according to the sufficient conditions in (21) (i.e., a semi-global result). The result in (20) can now be obtained from (35). Based on the definition of $y(t)$, the result in (35) indicates that $e_{1}(t), e_{2}(t) \in \mathcal{L}_{\infty}$ in $\mathcal{S}$. Given that $e_{1}(t), e_{2}(t), q_{d}(t), \dot{q}_{d}(t) \in \mathcal{L}_{\infty}$ in $\mathcal{S},(8),(13)$, and (9) indicate that $q(t), \dot{q}(t), V(t) \in \mathcal{L}_{\infty}$ in $\mathcal{S}$.

\section{EXPERIMENT RESULTS}

Experiments were conducted on a testbed which consists of a custom computer controlled stimulation circuit and a modified leg extension machine (LEM) depicted in Fig. 1. The LEM was modified to include optical encoders to measure the knee angle and a mechanical stop to prevent knee hyperextension. The encoder has a resolution of $0.018^{\circ}$ with a frequency response of $150 \mathrm{kHz}$. The LEM allows seating adjustments to ensure the rotation of the knee is about the encoder axis. Bipolar self-adhesive neuromuscular stimulation electrodes were placed over the distal-medial and proximal-lateral portion of the quadriceps femoris muscle group of the volunteers. The electrodes were connected to a custom stimulation circuitry which generates a fixed pulsewidth and a user-specified constant frequency stimulation train. Specifically, a nonsinusoidal square wave (pulse train) with constant pulsewidth and constant frequency was used.

Two sets of experiments were performed on nonimpaired male and female subjects (as in our previous study in [18]) with age ranges of 20 to 35 years, with written informed consent as approved by the Institutional Review Board at the University of Florida. Support for our selection of a noninjured population for this study is based on the fact that the electrical stimulation responses of nonimpaired subjects have been reported as similar to paraplegic subjects' responses [10], [11], [15], [54]. The objective of the first set of experiments was to characterize the input delay with respect to voltage (i.e., to investigate the input delay variation with respect to stimulation voltage). The other input delay dependencies such as stimulation frequency or pulsewidth are not reported as in the controller performance trials only stimulation voltage was varied while the stimulation frequency and pulsewidth were kept constant. Also, the delay dependencies on other stimulation parameters and factors such as fatigue are beyond the scope of this paper. The objective of the second set of experiments was to enable the lower leg to follow a desired continuous angular trajectory. The results in both experiments are examined through statistical analysis: Analysis of variance (ANOVA) method and Student's t-test were employed. To ensure that the collected data satisfies the assumptions of these tests, proper measures and protocols were followed. Specifically, subjects were unaware of the control method and the voltage factor being implemented, these results obtained were not disclosed to the subject, and the protocols were implemented in random order. These measures ensure that the data samples are independent. For, each method, the data samples were checked for normal distribution. Also, the data collected in the first set of experiments was checked for equality in variances. Since a paired t-test was employed for the second set of experiments, an "equality in variance test" is not required.

\section{A. Experiments: Input Delay Characterization}

Experiments were conducted to characterize input delay in healthy individuals during NMES. The EMD was measured as the difference between the time when voltage is applied at the electrode sites on the skin and the time when the angle encoder detects the first leg movement. Although in [21], [22], and [55] the EMD is measured as the time lag between the onset of muscle electrical activity (electromyogram, EMG) and tension development in human muscle, the method employed in this study is practical for use during NMES since firstly, significant (and sometimes questioned) filtering efforts are required to reliably measure EMG signal during NMES; secondly, the employed method accounts for total time course taken by the applied voltage at the electrode-skin interface to the observation of leg movement (analogous to muscle force production). The delay between applied voltage and muscle response is most valuable when design and control of NMES protocols is the goal. Note, due to the different method employed to measure EMD and particular type of muscle contraction considered in experiments, the measured EMD values can be or are different from the values reported in the literature. ${ }^{1}$

The input delay values were measured for ten healthy individuals (nine male and one female; age: 20 to 35 years). Tests on each individual were performed to investigate changes in the EMD as a result of variable voltage levels with fixed pulsewidth and frequency (i.e., the same conditions for the subsequent amplitude modulated control implementation). Before the start of experiments, the subject was instructed to relax to avoid voluntary leg motion. The threshold voltage was measured for each subject which can be defined as the minimum applied voltage that produces a movement large enough to be detected by the angle encoder. This measurement was performed by applying a constant input voltage, beginning at $10 \mathrm{~V}$ and increasing the voltage slightly until movement was detected. The frequency was kept at $30 \mathrm{~Hz}$ and the pulsewidth was kept at $100 \mu \mathrm{s}$. Four experiments were performed for different voltages (threshold voltage + additional voltage), where additional voltage was varied between 5 and $20 \mathrm{~V}$ in increments of $5 \mathrm{~V}$. Also, note that the selection of pulsewidth (i.e., $100 \mu \mathrm{s}$ ) could be changed to yield different results. The current study characterizes EMD during NMES for a limited range of voltages (5-20 V above a subject's threshold voltage). It was observed that during leg extension experiments the applied voltage did not go above any subject's threshold voltage by $20 \mathrm{~V}$; therefore, the voltage in the first set of experiments was kept within this range. However, for a complete EMD characterization study a wider range of voltages needs to be investigated.

The test consisted of measuring the input delay of the subject's muscle for three 0.2-s impulses, each $5 \mathrm{~s}$ apart. Each impulse imparted a constant voltage (threshold voltage + additional voltage) to the muscle. The 5-s time separation between the impulses allowed the subjects to voluntarily bring their leg back to the rest position. Fig. 2 shows the typical EMD during NMES (as a representative example). Table I indicates the summarized input delay variations with respect to varying voltage.

\footnotetext{
${ }^{1}$ The reported EMD values vary in the range of 30 and $100 \mathrm{~ms}$ [22] due to different methods and type of muscle contraction considered; and measured EMD values in this paper do fall in this range.
} 


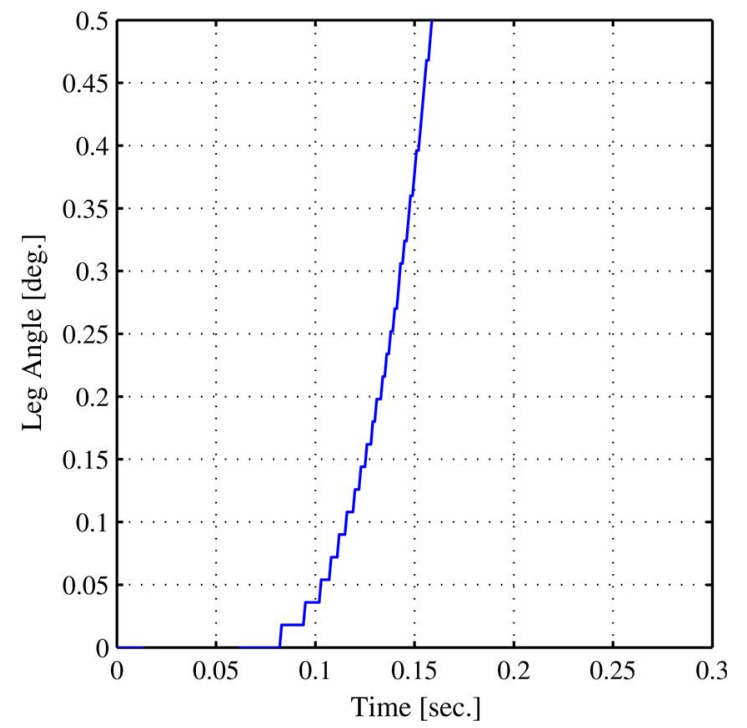

Fig. 2. Typical input delay during NMES. Voltage is applied at $\mathrm{t}=0$ but leg angle starts rising around $80 \mathrm{~ms}$.

TABLE I

SumMarized INPUT Delay VAlues of Healthy INDIVIDUAL ACROSS Different Stimulation Parameters. Delay Values $(\tau)$ Are Shown in Seconds. Voltages Shown in Column 3 Are Added Voltages to THRESHOLD VOLTAGE

\begin{tabular}{|c|c|c|c|c|c|}
\hline Subject & Voltage [V] & $\tau_{1}$ & $\tau_{2}$ & $\tau_{3}$ & Avg. $\tau$ \\
\hline \multirow[t]{4}{*}{$\mathbf{A}$} & 5 & 0.081 & 0.061 & 0.061 & 0.068 \\
\hline & 10 & 0.069 & 0.053 & 0.073 & 0.065 \\
\hline & 15 & 0.068 & 0.079 & 0.087 & 0.078 \\
\hline & 20 & 0.082 & 0.084 & 0.059 & 0.075 \\
\hline \multirow[t]{4}{*}{ B } & 5 & 0.067 & 0.063 & 0.053 & 0.061 \\
\hline & 10 & 0.085 & 0.072 & 0.091 & 0.083 \\
\hline & 15 & 0.06 & 0.054 & 0.042 & 0.052 \\
\hline & 20 & 0.061 & 0.06 & 0.06 & 0.060 \\
\hline \multirow[t]{4}{*}{$\mathbf{C}$} & 5 & 0.073 & 0.084 & 0.07 & 0.076 \\
\hline & 10 & 0.077 & 0.065 & 0.062 & 0.068 \\
\hline & 15 & 0.06 & 0.08 & 0.05 & 0.063 \\
\hline & 20 & 0.076 & 0.07 & 0.074 & 0.073 \\
\hline \multirow[t]{4}{*}{ D } & 5 & 0.089 & 0.074 & 0.096 & 0.086 \\
\hline & 10 & 0.088 & 0.105 & 0.084 & 0.092 \\
\hline & 15 & 0.069 & 0.063 & 0.073 & 0.068 \\
\hline & 20 & 0.067 & 0.07 & 0.079 & 0.072 \\
\hline \multirow[t]{4}{*}{$\mathbf{E}$} & 5 & 0.068 & 0.07 & 0.083 & 0.074 \\
\hline & 10 & 0.067 & 0.087 & 0.08 & 0.078 \\
\hline & 15 & 0.06 & 0.059 & 0.08 & 0.066 \\
\hline & 20 & 0.056 & 0.073 & 0.075 & 0.068 \\
\hline \multirow[t]{4}{*}{$\mathbf{F}$} & 5 & 0.075 & 0.08 & 0.082 & 0.079 \\
\hline & 10 & 0.065 & 0.069 & 0.072 & 0.069 \\
\hline & 15 & 0.074 & 0.079 & 0.08 & 0.078 \\
\hline & 20 & 0.081 & 0.059 & 0.06 & 0.067 \\
\hline \multirow[t]{4}{*}{$\mathbf{G}$} & 5 & 0.082 & 0.066 & 0.073 & 0.074 \\
\hline & 10 & 0.092 & 0.073 & 0.101 & 0.089 \\
\hline & 15 & 0.061 & 0.076 & 0.057 & 0.065 \\
\hline & 20 & 0.071 & 0.069 & 0.096 & 0.079 \\
\hline \multirow[t]{4}{*}{$\mathbf{H}$} & 5 & 0.074 & 0.067 & 0.09 & 0.077 \\
\hline & 10 & 0.081 & 0.059 & 0.092 & 0.077 \\
\hline & 15 & 0.044 & 0.058 & 0.07 & 0.057 \\
\hline & 20 & 0.057 & 0.047 & 0.044 & 0.049 \\
\hline \multirow[t]{4}{*}{ I } & 5 & 0.076 & 0.061 & 0.067 & 0.068 \\
\hline & 10 & 0.073 & 0.074 & 0.071 & 0.073 \\
\hline & 15 & 0.056 & 0.054 & 0.054 & 0.055 \\
\hline & 20 & 0.08 & 0.072 & 0.061 & 0.071 \\
\hline \multirow[t]{4}{*}{$\mathbf{J}$} & 5 & 0.074 & 0.118 & 0.107 & 0.100 \\
\hline & 10 & 0.061 & 0.059 & 0.071 & 0.064 \\
\hline & 15 & 0.082 & 0.056 & 0.106 & 0.081 \\
\hline & 20 & 0.058 & 0.062 & 0.067 & 0.062 \\
\hline
\end{tabular}

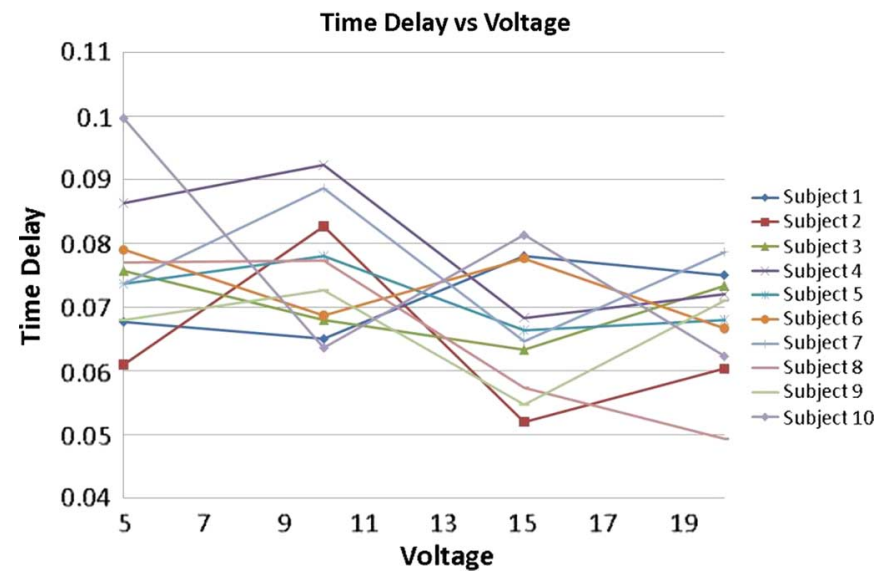

Fig. 3. Average input delay values across different voltages and ten subjects.

ANOVA tests were performed to determine the statistical significance between the means of data groups. Since one of the ANOVA test's assumption requires that the data samples belong to a normal distribution population, a Shapiro-Wilk test was employed. Also, to ensure that the data satisfies the equal variance test, a Levene's test was utilized. P-values greater than 0.05 were obtained in both tests which indicate that the data samples have equal variance and belong to a normally distributed population. The ANOVA results of the voltage experiments (see Fig. 3) showed no significant difference between stimulation voltage and electromechanical delay $(P-$ value $=0.072)$. These findings indicate that Assumption 5 is reasonable since there is no statistical differences in the EMD across subjects due to amplitude modulation.

\section{B. Experiments: Control Results}

The control objective of the second set of experiments was to track a continuous constant period ( $2 \mathrm{~s}$ ) sinusoidal trajectory. Three healthy males and one female (age 20-28 years) were chosen as the test subjects. For each session, a pretrial test was performed on each volunteer to find: 1) the appropriate initial voltage for the controller to reduce the initial transient error; 2) minimum or threshold voltage to be added to the computed controller voltage; and 3) maximum or saturation voltage to keep the leg movement within mechanical constraints (the maximum knee angle is also constrained by mechanical stop to avoid hyperextension). After the pretrial tests, the input delay value was measured for each subject. Input delay for each healthy individual was measured as a difference between the time when the voltage is applied and the time when first leg movement is observed. The measured delay value was utilized for implementing the new controller ( $P D$ controller with delay compensation) and throughout the duration of trials, the same respective measured delay value was used for each subject. Each subject participated in two to four trials of a 20 -s duration for each controller, depending on volunteer availability, where a rest period of 5 min was provided between the experimental trials. A frequency of $30 \mathrm{~Hz}$ and $400 \mu$ s pulsewidth were used for stimulation while the voltage was modulated. The choice of these stimulation parameters is within the ranges typically reported during NMES studies [56]. Also, note that a different pulsewidth (e.g., 
TABLE II

Comparison of Experimental Results Between Traditional PD CONTROller and PD CONTROLler With Delay Compensation. * INDiCATES

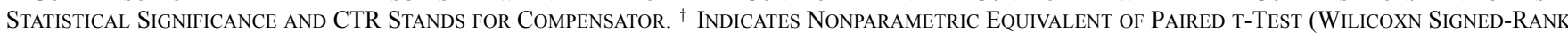
Test) Was Performed. NonParametric and Student's t-Tests Were Employed by ObTaining Mean of Best Two Results in Each CaSe

\begin{tabular}{|c|c|c|c|c|c|c|c|c|c|}
\hline \multirow[t]{2}{*}{ Subject } & \multirow{2}{*}{$\begin{array}{l}\text { EMD } \\
\text { [Sec.] }\end{array}$} & \multicolumn{2}{|c|}{ RMS Error } & \multicolumn{2}{|c|}{ Mean Voltage [V] } & \multicolumn{2}{|c|}{ Max. SSE } & \multicolumn{2}{|c|}{ Max. Voltage [V] } \\
\hline & & PD & PD+CTR & PD & PD+CTR & PD & PD+CTR & PD & PD+CTR \\
\hline \multirow[t]{2}{*}{$\mathbf{A}$} & \multirow[t]{2}{*}{0.075} & 4.48 & 5.26 & 31.49 & 33.18 & 11.84 & 11.51 & 42.95 & 42.02 \\
\hline & & 7.63 & 3.52 & 29.3 & 32.26 & 20.41 & 9.04 & 50 & 44.38 \\
\hline \multirow[t]{2}{*}{ B } & \multirow[t]{2}{*}{0.08} & 8.48 & 6.35 & 20.93 & 22.65 & 25.78 & 10.72 & 45.1 & 26.51 \\
\hline & & 6.54 & 5.96 & 24.72 & 22.93 & 10.79 & 9.611 & 31.28 & 27.43 \\
\hline \multirow[t]{2}{*}{ C } & \multirow[t]{2}{*}{0.07} & 3.11 & 2.85 & 25.58 & 26.17 & 12.84 & 5.68 & 43.68 & 38.8 \\
\hline & & 5.91 & 2.61 & 23.65 & 27.6 & 16.66 & 5.6 & 49.33 & 36.7 \\
\hline \multirow[t]{2}{*}{$\overline{\mathbf{D}}$} & \multirow{2}{*}{0.085} & 2.35 & 2.03 & 20.04 & 20.45 & 4.46 & 4.32 & 27.71 & 28.26 \\
\hline & & 3.73 & 2.54 & 21.94 & 21.68 & 10.99 & 4.93 & 30 & 29.49 \\
\hline \multirow[t]{2}{*}{$\mathbf{E}$} & \multirow[t]{2}{*}{0.062} & 6.278 & 3.8 & 19.81 & 21.09 & 18.72 & 10.11 & 30 & 30 \\
\hline & & 4.45 & 4.17 & 21.64 & 22.07 & 9.27 & 9.8116 & 29.7 & 30 \\
\hline \multicolumn{2}{|c|}{ Mean } & 5.30 & 3.91 & 23.91 & 25.01 & 14.18 & 8.13 & 37.98 & 33.36 \\
\hline \multicolumn{2}{|c|}{ p-value } & \multicolumn{2}{|c|}{$0.005^{*}$} & \multicolumn{2}{|c|}{0.05} & \multicolumn{2}{|c|}{$0.022 * \dagger$} & \multicolumn{2}{|c|}{0.058} \\
\hline
\end{tabular}

$100 \mu \mathrm{s}$ ) could have been utilized. Importantly, the impact of altering pulsewidths on muscle behavior is predictable (based on strength-duration curves) across different voltages and frequencies including those chosen for the current study [57], [58]. This means a different choice of pulsewidth would not have altered the findings reported below.

The main goal of the experiments was to quantify the added value of the delay compensation terms. Specifically, a traditional PD controller and the developed PD controller with the added delay compensation (identified as $\mathrm{PD}+\mathrm{CTR}$ in Table II) were randomly implemented to enable the lower shank to track a continuous constant period ( $2 \mathrm{~s})$ sinusoidal trajectory. The experimental results obtained for each controller are summarized in Table II. The table shows the best two results (results with minimum rms errors out of all trials) obtained from each controller and subject.

Remark 2: Since the performance of a controller can depend on control gain tuning, each controller was tested across a range of control gain values. Each subject participated in two to four trials with a 20 -s duration for each controller. The number of trials for each controller is limited and varies due to increasing discomfort that arises due to rapid muscle fatigue during NMES. Due to these reasons, it was not possible to compare the average of each controller results across all subjects and that is why only the best two control results for each controller were selected for statistical analysis.

Paired t-tests were also performed to confirm statistical significance in the mean differences of the rms errors, maximum steady-state errors (SSEs), rms voltages, and the maximum voltages. Since one of the t-test's assumption requires that the data samples belong to a normal distribution population, a ShapiroWilk test was employed. The results indicated that the data belongs to normal distribution except in the case of maximum SSEs obtained for PD + delay compensator. For this case, a nonparametric analogue of student's $t$ test was employed, i.e., maximum SSEs for PD controller and the developed controller were compared using the Wilcoxn signed-ranks test (for other cases, student's t-test was employed). The statistical analysis showed that the mean differences in the rms errors and maximum SSEs were statistically significant while no statistical significance was found between mean voltages and maximum volt- ages. The mean rms error of $3.91^{\circ}$ obtained with the $P D$ controller with delay compensation was lower than the rms error of $5.30^{\circ}$ obtained with the PD controller. Also, the mean maximum SSE and the mean maximum voltage obtained with the $P D$ controller with delay compensation were lower than those obtained with the traditional PD controller. The respective p-values obtained from the $\mathrm{t}$-test and nonparametric $\mathrm{t}$-test are given in the Table II. These tests were employed by obtaining mean of the best two results in each case. The actual leg angle, error, and voltage plots obtained from subject $\mathrm{C}$ (as a representative example) for the PD controller with delay compensation and the PD controller are shown in Figs. 4 and 5, respectively. Although no statistical significance was found between the mean maximum voltages of both controllers, it is interesting to note that the mean maximum voltage across the subjects was lower in the developed controller, where the difference was more than $4 \mathrm{~V}$. The statistically significant reduced rms error and the difference of more than $6^{\circ}$ in the maximum SSEs suggest better performance can be obtained through the new controller. In this study, a constant 2 -s desired trajectory was chosen; different desired trajectories may produce different responses (c.f. [18] and [17]) for both controllers examined.

These results also show robustness of the controller with respect to variations in input delay values, as the delay value for a subject can vary during the length of the trial or across different trials due to fatigue (c.f. [21] and [55] and references therein). The fact that the control gain $B$ was chosen without the knowledge of the ratio $\Omega(q, \dot{q}) / J$ (the ratio contains unknown muscle force-length and muscle force-velocity relationships, and moment arm; see Section II) shows that the gain condition in Remark 1 is sufficient but not necessary. Also, the results depict the robustness of the controller with respect to uncertainty in the ratio $\Omega(q, \dot{q}) / J$, since acceptable performance was obtained with different control gains B (all of the best two trials of the new controller utilized different control gain B for every but one subject).

\section{CONCLUSION}

A predictor-based NMES controller is developed to compensate for EMD in human skeletal muscle. A Lyapunov-based stability analysis is utilized to prove uniformly ultimately bounded 


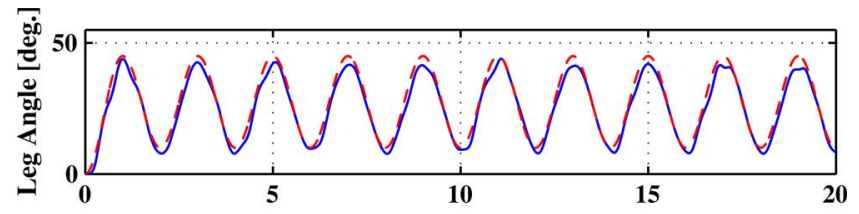

Time [sec.]
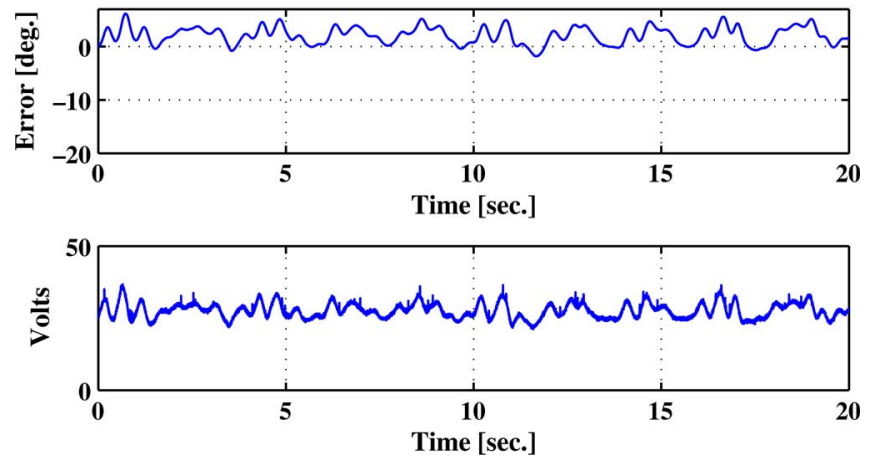

Fig. 4. Top plot: Actual limb trajectory of subject (solid line) versus desired trajectory (dashed line) obtained with PD controller with delay compensation. Middle plot: Tracking error (desired angle minus actual angle) of subject's leg. Bottom plot: Computed voltage of PD controller with delay compensation during knee joint tracking.
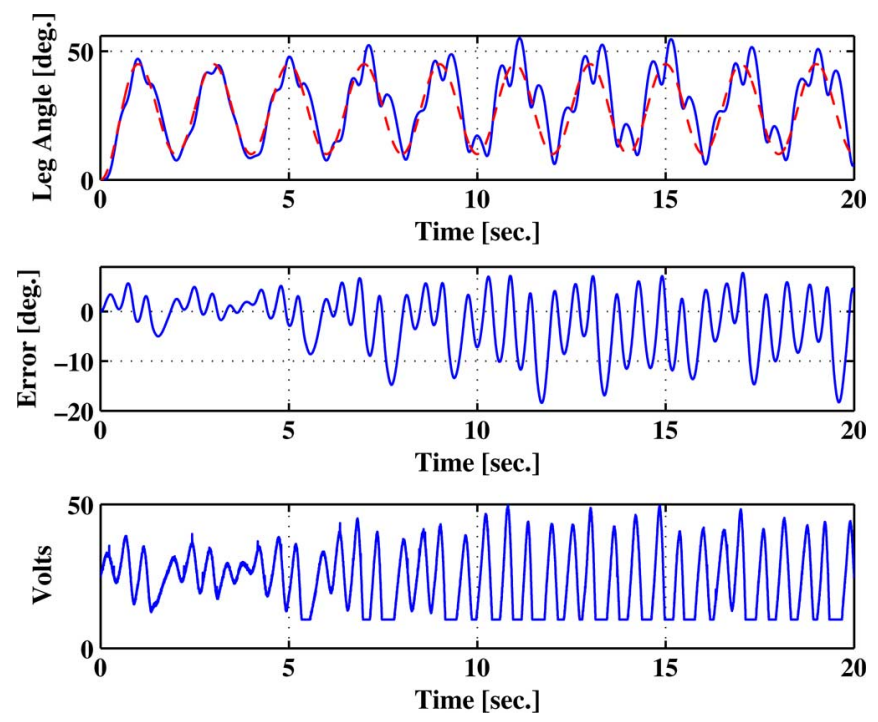

Fig. 5. Top plot: Actual limb trajectory of subject (solid line) versus desired trajectory (dashed line) input, obtained with traditional PD controller. Middle plot: Tracking error (desired angle minus actual angle) of subject's leg. Bottom plot: Computed PD voltage during knee joint tracking. Note that voltage saturates at user-defined set lower voltage threshold of $10 \mathrm{~V}$.

tracking for an uncertain nonlinear musculoskeletal system. The result is based on a limiting assumption that the EMD is constant. In practice, EMD is a complex phenomena that depends on the initial muscle length/joint angle, and varies as the muscle fatigues; however, the experimental results in this paper (for nonfatigued muscle) indicate that the EMD is statistically not different across the tested group of individuals and for multiple tests. This result justifies the assumption of a constant EMD (for muscle that does not transition to a fatigued state). The experimental outcomes indicate statistical improvement in the tracking error when delay compensation is included and also illustrates the robustness of the controller in the presence of some uncertainty and potential variability in the delay. Future efforts will focus on the development of controllers that account for variable and/or an unknown input delay, a currently open theoretical problem for uncertain nonlinear systems. The development of such controllers would provide additional robustness to changes in the EMD potentially caused by fatigue and other factors, which is especially important since muscle fatigues more quickly during NMES than through normal physiological processes. Also, the current study implicitly includes the effects of phase delay caused by the muscle contraction dynamics. This delay was discussed in detail in [23]. Since the current paper's scope is to examine a control method that compensates for EMD, future studies are required to highlight the role of EMD and phase delay caused by the active muscle contractile element.

\section{REFERENCES}

[1] P. H. Peckham and J. S. Knutson, "Functional electrical stimulation for neuromuscular applications," Annu. Rev. Biomed. Eng., vol. 7, pp. $327-360,2005$.

[2] J. J. Abbas and H. J. Chizeck, "Feedback control of coronal plane hip angle in paraplegic subjects using functional neuromuscular stimulation," IEEE Trans. Biomed. Eng., vol. 38, no. 7, pp. 687-698, Jul. 1991.

[3] J. J. Abbas and H. J. Chizeck, "Neural network control of functional neuromuscular stimulation systems: Computer simulation studies," IEEE Trans. Biomed. Eng., vol. 42, no. 11, pp. 1117-1127, Nov. 1995.

[4] N. Lan, P. E. Crago, and H. J. Chizeck, "Control of end-point forces of a multijoint limb by functional neuromuscular stimulation," IEEE Trans. Biomed. Eng., vol. 38, no. 10, pp. 953-965, Oct. 1991.

[5] T. Schauer, N. O. Negard, F. Previdi, K. J. Hunt, M. H. Fraser, E. Ferchland, and J. Raisch, "Online identification and nonlinear control of the electrically stimulated quadriceps muscle," Contr. Eng. Pract., vol. 13, pp. 1207-1219, 2005.

[6] A. H. Vette, K. Masani, and M. R. Popovic, "Implementation of a physiologically identified PD feedback controller for regulating the active ankle torque during quiet stance," IEEE Trans. Neural Syst. Rehabil. Eng., vol. 15, no. 2, pp. 235-243, Jun. 2007.

[7] K. Hunt and M. Munih, "Feedback control of unsupported standing in paraplegia-Part 1: Optimal control approach," IEEE Trans. Rehabil. Eng., vol. 5, no. 2, pp. 331-340, Jun. 1997.

[8] F. Previdi, M. Ferrarin, S. Savaresi, and S. Bittanti, "Gain scheduling control of functional electrical stimulation for assisted standing up and sitting down in paraplegia: A simulation study," Int. J. Adapt Contr. Signal Process., vol. 19, pp. 327-338, 2005.

[9] H. Gollee, K. Hunt, and D. Wood, "New results in feedback control of unsupported standing in paraplegia," IEEE Trans. Neural Syst. Rehabil. Eng., vol. 12, no. 1, pp. 73-91, Mar. 2004.

[10] G.-C. Chang, J.-J. Lub, G.-D. Liao, J.-S. Lai, C.-K. Cheng, B.-L. Kuo, and T.-S. Kuo, "A neuro-control system for the knee joint position control with quadriceps stimulation," IEEE Trans. Rehabil. Eng., vol. 5, no. 1, pp. 2-11, Mar. 1997.

[11] K. Kurosawa, R. Futami, T. Watanabe, and N. Hoshimiya, "Joint angle control by FES using a feedback error learning controller," IEEE Trans. Neural Syst. Rehabil. Eng., vol. 13, no. 4, pp. 359-371, Dec. 2005.

[12] S. Kim, M. Fairchild, A. Iarkov, J. Abbas, and R. Jung, "Adaptive control for neuromuscular stimulation-assisted movement therapy in a rodent model," IEEE Trans. Biomed. Eng., vol. 56, no. 3, pp. 452-461, Mar. 2008

[13] A. Ajoudani and A. Erfanian, "A neuro-sliding-mode control with adaptive modeling of uncertainty for control of movement in paralyzed limbs using functional electrical stimulation," IEEE Trans. Biomed. Eng., vol. 56, no. 7, pp. 1771-1780, Jul. 2009.

[14] J. Lujan and P. Crago, "Automated optimal coordination of multiple-DOF neuromuscular actions in feedforward neuroprostheses," IEEE Trans. Biomed. Eng., vol. 56, no. 1, pp. 179-187, Jan. 2009.

[15] S. Jezernik, R. Wassink, and T. Keller, "Sliding mode closed-loop control of FES: Controlling the shank movement," IEEE Trans. Biomed. Eng., vol. 51, no. 2, pp. 263-272, Feb. 2004.

[16] M. Ebrahimpour and A. Erfanian, "Comments on "sliding mode closed-loop control of FES: Controlling the shank movement," IEEE Trans. Biomed. Eng., vol. 55, no. 12, p. 2842, Dec. 2008. 
[17] N. Sharma, C. Gregory, M. Johnson, and W. E. Dixon, "Closed-loop neural network-based NMES control for human limb tracking," IEEE Trans. Contr. Syst. Technol., to be published.

[18] N. Sharma, K. Stegath, C. M. Gregory, and W. E. Dixon, "Nonlinear neuromuscular electrical stimulation tracking control of a human limb," IEEE Trans. Neural Syst. Rehabil. Eng., vol. 17, no. 6, pp. 576-584, Dec. 2009.

[19] N. Sharma, P. M. Patre, C. Gregory, and W. Dixon, "Nonlinear control of NMES: Incorporating fatigue and calcium dynamics," in Proc. ASME Dyn. Syst. Contr. Conf., 2009.

[20] R. Riener and T. Fuhr, "Patient-driven control of FES-supported standing up: A simulation study," IEEE Trans. Rehabil. Eng., vol. 6, no. 1, pp. 113-124, Mar. 1998.

[21] S. Zhou, D. Lawson, W. Morrison, and I. Fairweather, "Electromechanical delay in isometric muscle contractions evoked by voluntary, reflex and electrical stimulation," Eur. J. Appl. Physiol. Occup. Physiol., vol. 70, no. 2, pp. 138-145, 1995.

[22] P. Cavanagh and P. Komi, "Electromechanical delay in human skeletal muscle under concentric and eccentric contractions," Eur. J. Appl. Physiol. Occup. Physiol., vol. 42, no. 3, pp. 159-163, 1979.

[23] K. Masani, A. Vette, N. Kawashima, and M. Popovic, "Neuromusculoskeletal torque-generation process has a large destabilizing effect on the control mechanism of quiet standing," J. Neurophysiol., vol. 100, no. 3 , p. $1465,2008$.

[24] A. Vette, K. Masani, and M. Popovic, "Neural-mechanical feedback control scheme can generate physiological ankle torque fluctuation during quiet standing: A comparative analysis of contributing torque components," in IEEE Int. Conf. Contr. Applicat., 2008, pp. 660-665, IEEE.

[25] K. Masani, A. H. Vette, and M. R. Popovic, "Controlling balance during quiet standing: Proportional and derivative controller generates preceding motor command to body sway position observed in experiments," Gait Posture vol. 23, no. 2, pp. 164-172, Feb. 2006 [Online]. Available: http://dx.doi.org/10.1016/j.gaitpost.2005.01.006

[26] O. M. Smith, "A controller to overcome deadtime," ISA J., vol. 6, pp. 28-33, 1959

[27] Z. Artstein, "Linear systems with delayed controls: A reduction," IEEE Trans. Automat. Contr., vol. 27, no. 4, pp. 869-879, Apr. 1982.

[28] A. Manitius and A. Olbrot, "Finite spectrum assignment problem for systems with delays," IEEE Trans. Automat. Contr., vol. 24, no. 4, pp. 541-552, Apr. 1979.

[29] W. Michiels, K. Engelborghs, P. Vansevenant, and D. Roose, "Continuous pole placement for delay equations," Automatica, vol. 38, no. 5, pp. 747-761, 2002.

[30] Y. Yildiz, A. Annaswamy, I. Kolmanovsky, and D. Yanakiev, “Adaptive posicast controller for time-delay systems with relative degree $\mathrm{n} *<=2$," Automatica, vol. 46, no. 2, pp. 279-289, 2010.

[31] Y. Yildiz, A. Annaswamy, D. Yanakiev, and I. Kolmanovsky, "Spark ignition engine fuel-to-air ratio control: An adaptive control approach," Contr. Eng. Pract., vol. 18, pp. 1369-1378, 2010.

[32] Y. Yildiz, A. M. Annaswamy, D. Yanakiev, and I. Kolmanovsky, "Spark-ignition-engine idle speed control: An adaptive control approach," IEEE Trans. Contr. Syst. Technol., no. 99, 2011.

[33] C. Kravaris and R. Wright, "Deadtime compensation for nonlinear processes," AIChE J., vol. 35, no. 9, pp. 1535-1542, 1989.

[34] M. Henson and D. Seborg, "Time delay compensation for nonlinear processes," Ind. Eng. Chem. Res., vol. 33, no. 6, pp. 1493-1500, 1994.

[35] J. Huang and F. Lewis, "Neural-network predictive control for nonlinear dynamic systems with time-delay," IEEE Trans. Neural Networks, vol. 14, no. 2, pp. 377-389, Mar. 2003.

[36] N. Chopra, M. Spong, and R. Lozano, "Synchronization of bilateral teleoperators with time delay," Automatica, vol. 44, no. 8, pp. $2142-2148,2008$

[37] F. Mazenc and P. Bliman, "Backstepping design for time-delay nonlinear systems," IEEE Trans. Automat. Contr., vol. 51, no. 1, pp. 149-154, Jan. 2006.

[38] M. Jankovic, "Control of cascade systems with time delay-The integral cross-term approach," in Proc. IEEE Conf. Decis. Control, Dec. 2006, pp. 2547-2552.

[39] F. Mazenc, S. Mondie, S. Niculescu, P. Conge, I. Lorraine, and F. Metz, "Global asymptotic stabilization for chains of integrators with a delay in the input," IEEE Trans. Automat. Contr., vol. 48, no. 1, pp. 57-63, Jan. 2003.

[40] F. Mazenc, S. Mondie, R. Francisco, P. Conge, I. Lorraine, and F. Metz, "Global asymptotic stabilization of feedforward systems with delay in the input," IEEE Trans. Automat. Contr., vol. 49, no. 5, pp. 844-850, 2003.
[41] R. Francisco, F. Mazenc, and S. Mondié, Global Asymptotic Stabilization of a PVTOL Aircraft Model With Delay in the Input. New York: Springer, 2007.

[42] F. Mazenc and S. Bowong, "Tracking trajectories of the cart-pendulum system," Automatica, vol. 39, no. 4, pp. 677-684, 2003.

[43] M. Krstic, "On compensating long actuator delays in nonlinear control," IEEE Trans. Automat. Contr., vol. 53, no. 7, pp. 1684-1688, Jul. 2008.

[44] M. Krstic, "Input delay compensation for forward complete and strictfeedforward nonlinear systems," IEEE Trans. Automat. Contr., vol. 55, no. 2, pp. 287-303, Feb. 2010.

[45] A. Ailon, R. Segev, and S. Arogeti, "A simple velocity-free controller for attitude regulation of a spacecraft with delayed feedback," IEEE Trans. Automat. Contr., vol. 49, no. 1, pp. 125-130, Jan. 2004.

[46] A. Ailon, "Asymptotic stability in a flexible-joint robot with model uncertainty and multiple time delays in feedback," J. Franklin Inst., vol. 341, no. 6, pp. 519-531, 2004.

[47] A. Ailon and M. Gil, "Stability analysis of a rigid robot with outputbased controller and time delay," Syst. Contr. Lett., vol. 40, no. 1, pp. 31-35, 2000.

[48] N. Sharma, S. Bhasin, Q. Wang, and W. E. Dixon, "Predictor-based control for an uncertain Euler-Lagrange system with input delay," in Proc. Amer. Control Conf., 2010, pp. 1422-1427.

[49] M. Ferrarin and A. Pedotti, "The relationship between electrical stimulus and joint torque: A dynamic model," IEEE Trans. Rehabil. Eng., vol. 8, no. 3, pp. 342-352, Sep. 2000.

[50] J. L. Krevolin, M. G. Pandy, and J. C. Pearce, "Moment arm of the patellar tendon in the human knee," J. Biomech., vol. 37, pp. 785-788, 2004.

[51] W. L. Buford, Jr., F. M. Ivey, Jr., J. D. Malone, R. M. Patterson, G. L. Peare, D. K. Nguyen, and A. A. Stewart, "Muscle balance at the knee-Moment arms for the normal knee and the ACL-Minus knee," IEEE Trans. Rehabil. Eng., vol. 5, no. 4, pp. 367-379, Dec. 1997.

[52] R. Nathan and M. Tavi, "The influence of stimulation pulse frequency on the generation of joint moments in the upper limb," IEEE Trans. Biomed. Eng., vol. 37, no. 2, pp. 317-322, Feb. 1990.

[53] T. Watanabe, R. Futami, N. Hoshimiya, and Y. Handa, "An approach to a muscle model with a stimulus frequency-force relationship for FES applications," IEEE Trans. Rehabil. Eng., vol. 7, no. 1, pp. 12-17, Mar. 1999.

[54] J. Hausdorff and W. Durfee, "Open-loop position control of the knee joint using electrical stimulation of the quadriceps and hamstrings," Med. Biol. Eng. Comput., vol. 29, pp. 269-280, 1991.

[55] S. Zhou, M. McKenna, D. Lawson, W. Morrison, and I. Fairweather, "Effects of fatigue and sprint training on electromechanical delay of knee extensor muscles," Eur. J. Appl. Physiol. Occup. Physiol., vol. 72, no. 5, pp. 410-416, 1996.

[56] R. Riener and J. Quintern, Biomechanics and Neural Control of Posture and Movement, J. Winters and P. E. Crago, Eds. New York: SpringerVerlag, 2000.

[57] C. M. Gregory, W. E. Dixon, and C. S. Bickel, "Impact of varying pulse frequency and duration on muscle torque production and fatigue," Muscle Nerve, vol. 35, no. 4, pp. 504-509, 2007.

[58] C. M. Gregory, C. S. Bickel, N. Sharma, and W. E. Dixon, "Comparing the force- and excursion-frequency relationships in human skeletal muscle," Muscle Nerve vol. 38, no. 6, pp. 1627-1629, Dec. 2008 [Online]. Available: http://dx.doi.org/10.1002/mus.21161

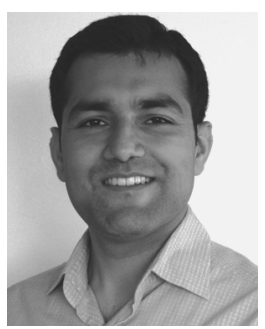

Nitin Sharma received the Ph.D. degree from the Department of Mechanical and Aerospace Engineering, University of Florida, Gainesville, in 2010.

Currently, he is an Alberta Innovates-Health Solutions postdoctoral Fellow in the Department of Physiology at the University of Alberta, Edmonton, Canada. His research interests include intelligent and robust control of functional electrical stimulation (FES), modeling, optimization, and control of FES-elicited walking, and control of uncertain nonlinear systems with input and state delays.

Dr. Sharma is a recipient of 2009 O. Hugo Schuck Award and Best Student Paper Award in Robotics at the 2009 ASME Dynamic Systems and Controls Conference. He was also a finalist for the Best Student Paper Award at the 2008 IEEE Multi-Conference on Systems and Control. 


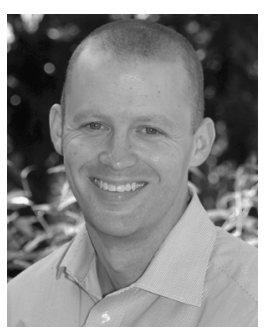

Chris M. Gregory received the Ph.D. degree in exercise physiology from the University of Georgia, Athens, in 2002, and completed subsequent postdoctoral training in muscle biology at the University of Florida, Gainesville.

$\mathrm{He}$ is currently an Assistant Professor at the Medical University of South Carolina and a Research Scientist at the Ralph H. Johnson VA Medical Center, Charleston, SC. His research focuses on the study of skeletal muscle form and function. During his career, he has published over 80 manuscripts, conference proceedings, book chapters and abstracts on topics including muscle physiology, biochemistry, exercise and electrical stimulation of skeletal muscle.

Dr. Gregory received an Associate Investigator Award and a Career Development Award (level II) from the Department of Veterans Affairs in 2005.

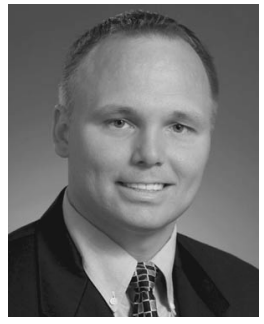

Warren E. Dixon (M'04) received the Ph.D. degree from the Department of Electrical and Computer Engineering, Clemson University, in 2000.

After completing his doctoral studies he was selected as a Eugene P. Wigner Fellow at Oak Ridge National Laboratory (ORNL). In 2004, he joined the faculty of the Mechanical and Aerospace Engineering Department, University of Florida. His main research interest has been the development and application of Lyapunov-based control techniques for uncertain nonlinear systems. He has published three books, an edited collection, six chapters, and over 200 refereed journal and conference papers.

Dr. Dixon's work has been recognized by the 2009 American Automatic Control Council (AACC) O. Hugo Schuck Award, 2006 IEEE Robotics and Automation Society (RAS) Early Academic Career Award, an NSF CAREER Award (2006-2011), 2004 DOE Outstanding Mentor Award, and the 2001 ORNL Early Career Award for Engineering Achievement. He serves as an Associate Editor for ASME Journal, Journal of Dynamic Systems, Measurement and Control, Automatica, IEEE Transactions on Systems Man and Cybernetics: Part B Cybernetics, International Journal of Robust and Nonlinear Control, and Journal of Robotics. 